\title{
Eintomo Brersilis \\ Sinopse dos Phasmatodea (Insecta) Descritos para o Brasil
}

\author{
Fernanda Figueiredo de Araujo \& André Rinaldo Senna Garraffoni
}

\author{
Universidade Federal dos Vales do Jequitinhonha e Mucuri, e-mail: figueiredofaraujo@gmail.com, garraffoni@gmail.com (Autor para \\ correspondência ${ }^{\bowtie}$ ).
}

EntomoBrasilis 5 (3): 232-237 (2012)

Resumo. A sistemática é a ciência que descreve, nomeia, classifica e determina as relações entre os seres vivos. Atualmente ela enfrenta alguns problemas como a falta de especialistas em alguns grupos e regiões pouco amostradas. Os fasmídeos ainda são pouco estudados no Brasil e não existe registro de especialistas na ordem, causando dessa forma, diversas dificuldades na identificação dos espécimes do táxon. Contudo, apesar desse baixo conhecimento é possível notar vários problemas taxonômicos e pouca literatura disponível. Com isso, este trabalho tem como objetivo reconhecer e listar as espécies de Phasmatodea originalmente descritos para o Brasil, além de tecer alguns comentários sobre o histórico taxonômico do grupo no país.

Palavras-chaves: Classificação; Fasmídeos; Salvador de Toledo Piza.

\section{Synopsis of Phasmatodea (Insecta) Described from Brazil}

Abstract. Systematics involves description, naming of taxa, classification and phylogenetics determination of evolutionary relationships among the living. The decline in taxonomists available and the lack of taxonomic expertise lead to the so-called taxonomical impediments. The diversity and distribution of the phasmids in Brazil is still little known as any taxonomist is available in the country. However, although this poor knowledge in the Brazilian phasmids, it is possible to observe many taxonomical problems, very few literatures available. Thus, in this paper we listed all species originally described from Brazil and pointed out some notes about the taxonomic history of the phasmids from Brazil.

Keywords: Classification; Phasmids; Salvador de Toledo Piza.

$\mathbf{E}$ studos sistemáticos e taxonômicos têm como alvo os táxons (SAVAGE 1995), os quais podem incluir desde uma única espécie a milhares de espécies. Geralmente, o desenvolvimento de um estudo taxonômico pode exigir o estudo de vários exemplares. Alguns destes estudos abrangem espécies de apenas uma região, assim algumas coleções taxonômicas tornam-se restritas para possíveis comparações e em diversos táxons, incluindo a maioria dos invertebrados, a proporção de espécies e especialistas é muito desigual (WHEeler 2007). Outro problema que taxonomia enfrenta é a falta de curadores e responsáveis por coleções preparados para arrecadar verbas e manter as coleções conservadas. Como em qualquer outra ciência, é necessário que novos trabalhos taxonômicos sejam realizados. Entretanto, necessita-se de forte apoio financeiro por parte dos governos e empenho intelectual dos pesquisadores para alavancar o desenvolvimento da taxonomia (CRISCI 2006).

O Brasil, possui cerca de $13 \%$ de toda a biota terrestre e por isso é considerado um país megadiverso (LeWINSOHN \& PRADO 2005). Porém, essa fauna é pouco conhecida (Lewinsohn et al. 2005) e estima-se que as espécies descritas até o presente sejam somente $6,67 \%$ do total. Este problema é decorrente de fatores como, existência de regiões pouco amostradas, reduzido número de inventários realizados que reflete diretamente no baixo número de exemplares depositados em museus e número insuficiente de pesquisadores (MARQUES \& LAMAS 2006), o que ocasiona uma dependência de pesquisadores estrangeiros, principalmente europeus.
Com aproximadamente 3000 espécies descritas em todo mundo (Отте \& BRock 2005), das quais 200 no Brasil (Zoмpro \& Domenico 2005), a ordem Phasmatodea ainda é pouco estudada, uma vez que, são encontradas diversas dificuldades na identificação dos espécimes, tais como: (i) descrições resumidas que fornecem poucas informações para a identificação das espécies; (ii) acentuado dimorfismo sexual que pode ocasionar a descrição de novas espécies a partir de coleta de indivíduos de diferentes sexos; (iii) variação intraespecífica, como tamanho, coloração e textura do corpo; (iv) espécies descritas a partir de um único espécime (macho, fêmea ou ninfa), geralmente mal preservado; (v) uso apenas de características morfológicas externas, não sendo relatado nada sobre as estruturas internas e a morfologia dos ovos; (vi) poucos trabalhos sistemáticos, juntamente com baixo número de coletas, envolvendo a ordem, e consequente falta de material depositado em museus, o que ocasiona a impossibilidade de trabalhos comparativos (CONLE \& Hennemann 2011). Dessa forma, o presente trabalho teve como objetivo reconhecer e listar as espécies pertencentes ao grupo dos Phasmatodea e originalmente descritos para o Brasil, além de tecer alguns comentários sobre o histórico taxonômico do grupo no país.

\section{MATERIAL E MÉTODOS}

A partir de um levantamento realizado através do banco de dados online Phasmida Species File (ВRоск et al. 2011) e do estudo de Zompro \& Domenico (2005), foi possível listar algumas espécies cujos holótipos estão depositados em museus e coleções pelo 
mundo, dentre eles os museus brasileiros ESLQTP (Escola Superior de Agricultura "Luiz de Queiroz", Coleção Toledo Piza, Piracicaba, São Paulo), MZSP (Museu de Zoologia da Universidade São Paulo, São Paulo), IBSP (Instituto Biológico de São Paulo, São Paulo) e MPEG (Museu Paraense Emílio Goeldi, Belém, Pará), e ICB- UFMG (Instituto de Ciências BiológicasUniversidade Federal de Minas Gerais), os europeus BMNH (British Museum of Natural History, Londres, Inglaterra), NHMW (Naturhistorisches Museum, Vienna, Áustria), MHNG (Museum d'histoire Naturelle, Genebra, Suíça), ETHZ (EidgenössischeTechnischeHochschuleZürich, Entomologisches Institut, Zurique, Suíça), OXUM (Oxford University Museum of Natural History, Oxford, Inglaterra), ZMHB (Zoologisches Museum del' Humboldt-Universitiit, Berlim, Alemanha), SMFD (Forschungsinstitut und Naturmuseum Senckenberg, Frankfurt amMain,Alemanha),SMNS(Staatliches MuseumfürNaturkunde, Stuttgart, Alemanha), ZMUH (Zoologisches Museum und Universität Hamburg, Hamburgo, Alemanha), MLUH (Martin Luther Universität Halle, Halle, Alemanha), SMTD (Staatliches Museum für Tierkunde, Dresden, Alemanha), ISNB (Institute Royal des Sciences Naturalles de Belgique, Bruxelas, Bélgica), MNHN (Museum National d'Histoire Naturelle, Paris, França), NHRS (Naturhistoriska Riksmuseet, Estocolmo, Suécia), MRSN (Museo Regionale Di Science Naturali, Turim, Itália), MIZ (Museum I Instytut Zoologii, Polskiej Akademii Nauk, Varsóvia, Polônia), MNMS (Museo Nacional de Ciencias Naturales, Madri, Espanha), MVMA (Museum Victoria, Melbourne, Austrália) e ANSP (Academy of Natural Sciences of Drexel University, Filadélfia, EUA).

\section{RESULTADOS E DISCUSSÃO}

No Brasil, foram descritas mais de 200 espécies de Phasmatodea (Brock et al. 2011) e no presente estudo conseguimos levantar informações de 174 espécies (Tabela 1). Foi possível averiguar que a maior parte dessas espécies foi descrita por pesquisadores estrangeiros e estão depositadas em diversos museus, principalmente europeus. Essas descrições foram realizadas, em sua maioria, entre as últimas décadas do século XVIII e o fim do século XX, com poucas descrições no século XXI para o Brasil. Além disso, foi possível constatar que, apenas 49 das mais de 200 espécies coletadas e descritas para o Brasil apresentavam material tipo depositado nos museus nacionais, o que equivale a aproximadamente $1 / 4$ do total de espécies, sendo que as demais espécies estão depositadas em museus estrangeiros (Tabela 1). Vale ressaltar que algumas espécies que apresentam materialtipo nos museus brasileiros não foram coletadas em território nacional, embora sejam consideradas no trabalho de Zoмpro \& Domenico (2005) e descartado no presente trabalho.

Foi constatado também que 45 das 49 espécies (representando mais de 90\%) com holótipos depositados em museus brasileiros foram descritas por um único pesquisador entre o período de 1936 e 1985 (Toledo Piza 1936a, 1936b, 1937, 1938a, 1938b, 1939, 1943, 1944, 1977, 1985a, 1985b). Assim, este pesquisador foi responsável por pouco mais de 20\% do total de espécies descritas para o Brasil. Após a sua morte, m 1988, poucos pesquisadores realizaram descrições de novas espécies no Brasil (Zompro \& ADIS 2001; Zompro 2004a, 2004b; Kumagai \& FonseCa 2009). Desta forma, pode-se observar diferentes momentos da expansão do conhecimento da ordem no país: um momento onde houve um grande número de descrições, e momentos de grandes pausas seguido por um lento retorno às atividades taxonômicas de Phasmatodea no Brasil.

O fato de existir poucos pesquisadores especialistas, falta de material tipo ou exemplares em estado de conservação ruim e escassa literatura especializada (que resulta, entre outros problemas, a falta de chaves de identificação) sobre a ordem no país pode ter causado alguns dos problemas taxonômicos observados no presente estudo. A de se destacar que boa parte das espécies originalmente descritas para o Brasil (55\% do total) foram sinonimizados ou mesmo transferidos de gênero (Tabela 1).

Tabela 1. Lista das espécies de fasmídeos descritos para o Brasil, bem como os museus as quais as séries tipos estão depositadas e seus status taxonômicos atual.

\begin{tabular}{|c|c|c|c|c|c|}
\hline Gênero & Espécie & Autor/ano & Localidade-tipo & Museu $^{*}$ & Status atual \\
\hline \multirow{3}{*}{ Abrachia } & A. borellii & Giglio-Tos, 1910 & Urucum, MT & MRSN & Baculum borellii \\
\hline & A. brevicornis & Kirby, 1889 & Teresópolis, RJ & BMNH & Baculum brevicornis \\
\hline & A. euterpinus & Westwood, 1859 & Brasil & BMNH & Canuleius euterpinus \\
\hline \multirow{4}{*}{ Acanthoderus } & A. libidinosus & Toledo Piza, 1943 & Mogi das Cruzes, SP & ESLQTP & Canuleius libidinosus \\
\hline & A. oryx & Westwood, 1859 & Brasil & ZMHB & Ocnophila oryx \\
\hline & A. scops & Kaup, 1871 & Brasil & SMFD, & Ocnophila scops \\
\hline & A. vigilans & Westwood, 1859 & Brasil & ZMHB & Pygirhynchus vigilans \\
\hline \multirow{2}{*}{ Anisomorpha } & A. dentata & Stål, 1875 & SC, Brasil & NHMW & Pseudophasma dentata \\
\hline & A. urucumana & Giglio-Tos, 1910 & Urucum, MT & MRSN & Urucumania urucumana \\
\hline \multirow{4}{*}{ Bacillus } & B. (Baculum) ramosum & Saussure, 1861 & Brasil & MHNG & Baculum ramosum \\
\hline & B. arampes & Kaup \& Heyden, 1871 & Brasil & SMFD & Heteronemia arampes \\
\hline & B. aurita & Burmeister, 1838 & Brasil & OXUM & Otocrania aurita \\
\hline & B. culmus & Bates, 1865 & Brasil & OXUM & Bacteria culmus \\
\hline \multirow{8}{*}{ Bacteria } & B. emortualis & Saussure, 1859 & BA, Brasil & MHNG & Heteronemia emortualis \\
\hline & B. amazonica & Bates, 1865 & AM, Brasil & OXUM & Heteronemia mazônica \\
\hline & B. longimana & Saussure, 1859 & BA, Brasil & MHNG & Baculum longimanus \\
\hline & B. muricata & Burmeister, 1838 & PA, Brasil & ZMHB & Phanocloidea muricata \\
\hline & B. satyr & Redtenbacher, 1908 & Brasil & SMTD & Phanocloidea satyr \\
\hline & B. tuberculata & Toledo Piza, 1936a & São Paulo, SP & MZSP & $\begin{array}{c}\text { Cladomorphus phyllinus } \\
\text { (Gray, 1835) }\end{array}$ \\
\hline & B. waehneri & Günther, 1940 & Manaus, AM & SMTD & Aplopocranidium waehneri \\
\hline & B. dentipes & Redtenbacher, 1908 & Teresópolis, RJ & NHMW & Cladoxerus dentipes \\
\hline
\end{tabular}




\begin{tabular}{|c|c|c|c|c|c|}
\hline Gênero & Espécie & Autor/ano & Localidade-tipo & Museu* $^{*}$ & Status atual \\
\hline \multirow{4}{*}{ Bactridium } & B. grande & Rehn, 1920 & SC, Brasil & ANSP & Phobaeticus serratipes \\
\hline & B. maximum & Redtenbacher, 1908 & Brasil & NHMW & Heteronemia maximus \\
\hline & B. pausispinosum & Redtenbacher, 1908 & RJ, Brasil & NHMW & Heteronemia paucispinosus \\
\hline & B. imperialis & Redtenbacher, 1908 & Brasil & ZMHB & Otocrania imperalis \\
\hline \multirow{2}{*}{ Bostra } & B. magnifica & Redtenbacher, 1908 & Brasil & SMNS & Bostra magnifica \\
\hline & B. pruinosa & Redtenbacher, 1908 & Brasil & NHMW & Bostra pruinosa \\
\hline \multirow[t]{9}{*}{ Brizoides } & B. flavipennis & Redtenbacher, 1906 & Brasil & SMTD & Brizoides flavipennis \\
\hline & B. affinis & Toledo Piza, 1936a & Alto da Serra, SP & MZSP & Canuleius affinis \\
\hline & C. bispinosus & Toledo Piza, 1936b & Jequié, BA & MZSP & Canuleius bispinosus \\
\hline & C. brevipes & Toledo Piza, 1936b & Alto da Serra, SP & IBSP & Canuleius brevipes \\
\hline & C. corallinus & Toledo Piza, 1936b & Alto da Serra, SP & MZSP & Canuleius corallinus \\
\hline & C. fischeri & Toledo Piza, 1936b & RJ, Brasil & MZSP & Canuleius fischeri \\
\hline & C. grandis & Toledo Piza, 1936b & Alto da Serra, SP & MZSP & Canuleius grandis \\
\hline & C. inermipes & Toledo Piza, 1944 & Ubatuba, SP & MZSP & Canuleius inermipes \\
\hline & C. inermis & Redtenbacher, 1906 & ES, Brasil & NHMW, & Canuleius inermis \\
\hline \multirow[t]{12}{*}{ Canuleius } & C. metzi & Redtenbacher, 1906 & SP, Brasil & ZMUH & Canuleius metzi \\
\hline & C. nudiceps & Redtenbacher, 1906 & SC, Brasil & NHMW & Canuleius nudiceps \\
\hline & C.pullus & Redtenbacher, 1906 & Teresópolis, ES & ISNB & Canuleius pullus \\
\hline & C. ubatubae & Toledo Piza, 1944 & Ubatuba, SP & MZSP & Canuleius ubatubae \\
\hline & C. similis & Redtenbacher, 1906 & Teresópolis, RJ & NHMW & Canuleius similis \\
\hline & C. vetus & Toledo Piza, 1936b & SC, Brasil & MZSP & Canuleius vetus \\
\hline & C. vigintiquatuorspinosus & Redtenbacher, 1906 & RJ, Brasil & NHMW & $\begin{array}{l}\text { Canuleius } \\
\text { vigintiquatuorspinosus }\end{array}$ \\
\hline & C. vigintispinosus & Redtenbacher, 1906 & RJ, Brasil & MNMS & $\begin{array}{c}\text { Canuleius } \\
\text { vigintispinosus }\end{array}$ \\
\hline & C. (Ceroys) albogranulatus & Toledo Piza, 1938b & Alto da Serra, SP & ESLQTP & $\begin{array}{l}\text { Ceroys (Ceroys) } \\
\text { albogranulatus }\end{array}$ \\
\hline & C. auritus & Toledo Piza, 1936b & SP, Brasil & MZSP & $\begin{array}{l}\text { Pygirhynchus toledopizai } \\
\text { (Zompro, 2004a) }\end{array}$ \\
\hline & C. (Miroceroys) brunneri & Toledo Piza, 1936b & Macaé, RJ & MZSP & $\begin{array}{l}\text { Ceroys (Miroceroys) } \\
\text { brunneri }\end{array}$ \\
\hline & C. (Ceroys) cristatus & Redtenbacher, 1906 & Brasil & NHMW & Ceroys (Ceroys) cristatus \\
\hline \multirow[t]{5}{*}{ Ceroys } & C. ignavus & Westwood, 1859 & Tapajos, AM & $\mathrm{BMNH}$ & Lobolibethra ignava \\
\hline & C. (Ceroys) multispinosum & Serville, 1838 & Brasil & OXUM & $\begin{array}{l}\text { Ceroys }(\text { Ceroys }) \\
\text { multispinosum }\end{array}$ \\
\hline & C. multispinosum & Toledo Piza, 1936b & Alto da Serra, SP & MZSP & Ceroys (Ceroys) spinosus \\
\hline & C. (Miroceroys) saevissimus & Westwood, 1859 & Brasil & $\mathrm{BMNH}$ & $\begin{array}{c}\text { Ceroys(Miroceroys) } \\
\text { saevissimus }\end{array}$ \\
\hline & C. (Ceroys) scaber & Toledo Piza, 1936b & Alto da Serra, SP & MZSP & Ceroys (Ceroys) scaber \\
\hline \multirow[b]{2}{*}{ Citrina } & C. modesta & Toledo Piza, 1985 & Serra do Navio, AP & MZSP & Cesaphasma modestum \\
\hline & C. ceratocephalus & Gray, 1835 & Brasil & OXUM & $\begin{array}{l}\text { Cladomorphus } \\
\text { ceratocephalus }\end{array}$ \\
\hline \multirow{3}{*}{ Cladomorphus } & C. trimariensis & $\begin{array}{c}\text { Kumagai \& Fonseca, } \\
2009\end{array}$ & Três Marias,MG & $\begin{array}{l}\text { ICB- } \\
\text { UFMG }\end{array}$ & Cladomorphus rimariensis \\
\hline & C.perfoliatus & Gray, 1835 & Brasil & $\mathrm{BMNH}$ & Ceroys Ceroys) perfoliatus \\
\hline & C. cryphaleus var. dentatus & Toledo Piza, 1938a & São Paulo, SP & ESLQTP & Baculum dentatum \\
\hline \multirow[t]{2}{*}{ Cladoxerus } & C. gracilis & $\begin{array}{c}\text { Le Peletier de Saint } \\
\text { Fargeau \& Serville, } \\
1827\end{array}$ & Brasil & $\mathrm{NE}$ & Cladoxerus gracilis \\
\hline & C.rubus & Saussure, 1861 & BA, Brasil & MHNG & Cladomorphus rubus \\
\hline Creoxylus & C.paradoxa & Kirby, 1904 & $\begin{array}{c}\text { Floresta de Santarém, } \\
\text { AM }\end{array}$ & $\mathrm{BMNH}$ & Creoxylus paradoxa \\
\hline \multirow{4}{*}{ Damasippus } & D. discoidalis & Redtenbacher, 1906 & Teresópolis, RJ & NHMW & Damasippus discoidalis \\
\hline & D. piceipennis & Redtenbacher, 1906 & MG, Brasil & ZMUH & Damasippus piceipennis \\
\hline & D. spatulatus & Toledo Piza, 1937 & Serra de Macaé, RJ & MZSP & Damasippus spatulatus \\
\hline & D. unilineatus & Redtenbacher, 1906 & Nova Friburgo, RJ & NHMW & Damasippus unilineatus \\
\hline Diapherodes & D. gibbosa & Burmeister, 1838 & Brasil & OXUM & Cranidium gibbosum \\
\hline \multirow{3}{*}{ Dilophocephalus } & D. paradiacanthoides & Toledo Piza, 1938b & Brasil & ESLQTP & $\begin{array}{l}\text { Paradiacantha } \\
\text { acanthocephala }\end{array}$ \\
\hline & D. agrion & Westwood, 1859 & Vila Nova, AM & BMNH & Dinelytron agrion \\
\hline & D. batesianum & Westwood, 1859 & AM, Brasil & OXUM & Damasippus batesianus \\
\hline
\end{tabular}




\begin{tabular}{|c|c|c|c|c|c|}
\hline Gênero & Espécie & Autor/ano & Localidade-tipo & Museu* & Status atual \\
\hline \multirow{3}{*}{ Dinelytron } & D. hipponax & Gray, 1835 & Brasil & $\mathrm{NE}$ & Dinelytron hipponax \\
\hline & D. shuckardi & Gray, 1835 & Brasil & $\mathrm{NE}$ & Dinelytron shuckardi \\
\hline & D. villosipes & Redtenbacher, 1906 & Brasil & MNHN & Dinelytron villosipes \\
\hline Dixippus & D. brasiliensis & Toledo Piza, 1938b & Brasil & ESLQTP & Hermagoras hosei \\
\hline Donusa & D. nigrovittata & Toledo Piza, 1939 & Salto Grande, SP & ESLQTP & Neophasma nigrovittata \\
\hline \multirow{4}{*}{ Dyme } & D. abnormis & $\begin{array}{c}\text { Brunner von } \\
\text { Wattenwyl, } 1907\end{array}$ & São Paulo, SP & ETHZ & Bacteria abnormis \\
\hline & D. brasiliensis & $\begin{array}{l}\text { Brunner von } \\
\text { Wattenwyl, } 1907\end{array}$ & Brasil & NHMW & Bacteria brasiliensis \\
\hline & D. ingenua & $\begin{array}{l}\text { Brunnervon } \\
\text { Wattenwyl, } 1907\end{array}$ & Brasil & NHMW & Canuleius euterpinus \\
\hline & D. parasanguinolenta & $\begin{array}{l}\text { Brunner von } \\
\text { Wattenwyl, } 1907\end{array}$ & Brasil & NHMW & $\begin{array}{c}\text { Bacteria } \\
\text { parasanguinolenta }\end{array}$ \\
\hline \multirow{2}{*}{ Echetlus } & E. evoneobertii & Zompro\& Adis, 2001 & $\begin{array}{l}\text { Boa Esperança do } \\
\text { Sul, SP }\end{array}$ & MZSP & Candovia evoneobertii \\
\hline & E. fulgens & Zompro, 2004b & Barra dos Bugres, MT & MPEG & Echetlus fulgens \\
\hline \multirow{2}{*}{ Gratidia } & G. debilis & Toledo Piza, 1938b & CE, Brasil & ESLQTP & Xiphophasma debilis \\
\hline & G. fragilis & Toledo Piza, 1938b & $\mathrm{CE}$, Brasil & ESLQTP & Xiphophasma fragilis \\
\hline Haplopus & H. eucnemis & Burmeister, 1838 & Brasil & ZMHB & Haplopus eucnemis \\
\hline \multirow{3}{*}{ Hirtuleius } & H. laeviceps & Stål, 1875 & Brasil & NHRS & Hirtuleius laeviceps \\
\hline & I. paulensis & Toledo Piza, 1944 & Ubatuba-SP & MZSP & Isagoras paulensis \\
\hline & I. metricus & Rehn, 1947 & Teffé, AM & ANSP & Isagoras metricus \\
\hline \multirow{4}{*}{ Isagoras } & I.nitidus & Redtenbacher, 1906- & Brasil & NHMW & Isagoras nitidus \\
\hline & I. plagiatus & Redtenbacher, 1906 & ES, Brasil & NHMW & Isagoras plagiatus \\
\hline & I. sauropterus & Rehn, 1947 & Teffé, AM & ANSP, & Isagoras sauropterus \\
\hline & I. tacanae & Günther, 1940 & Rio Tacana, AM & SMTD & Isagoras tacanae \\
\hline Libethra & L. unidentata & $\begin{array}{c}\text { Brunner von } \\
\text { Wattenwyl, 1907 }\end{array}$ & Brasil & NHMW & Libethra unidentata \\
\hline Mantis & M. lateralis & Fabricius, 1775 & Brasil & BMNH & $\begin{array}{c}\text { Prexaspes (Prexaspes) } \\
\text { lateralis }\end{array}$ \\
\hline \multirow{3}{*}{ Melophasma } & M. vermiculare & Redtenbacher, 1906 & ES, Brasil & NHMW & Melophasma vermiculare \\
\hline & M. armatum & Redtenbacher, 1906 & Brasil & SMTD & $\begin{array}{c}\text { Metriophasma } \\
\text { (Metriophasma) armatum }\end{array}$ \\
\hline & M. bubastes & Westwood, 1859 & Tapajos, AM & BMNH & Olinta bubastes \\
\hline \multirow{3}{*}{ Metriotes } & M. jurinei & Saussure, 1868 & Jurinei, AM & MHNG & Isagoras jurinei \\
\hline & M.pericles & Redtenbacher, 1906 & Brasil & NHMW & $\begin{array}{c}\text { Metriophasma } \\
\text { (Metriophasma) Péricles }\end{array}$ \\
\hline & M. santara & Westwood, 1859 & Santarem, AM & BMNH & Isagoras santara \\
\hline \multirow{2}{*}{ Miroceroys } & M. heymonsi & Toledo Piza, 1936b & Alto da Serra, SP & IBSP & $\begin{array}{c}\text { Ceroys (Miroceroys) } \\
\text { heymonsi }\end{array}$ \\
\hline & M. redtendebacheri & Toledo Piza, 1936b & Serra de Macaé, RJ & MZSP & $\begin{array}{l}\text { Ceroys (Miroceroys) } \\
\text { redtendebacheri }\end{array}$ \\
\hline \multirow{6}{*}{ Ocnophila } & O. capitata & $\begin{array}{c}\text { Brunner von } \\
\text { Wattenwyl, 1907 }\end{array}$ & SC, Brasil & ZMUH & Ocnophila capitata \\
\hline & O. cornuta & $\begin{array}{c}\text { Brunner von } \\
\text { Wattenwyl, } 1907\end{array}$ & MG, Brasil & NHMW & Ocnophila cornuta \\
\hline & O.fortior & $\begin{array}{c}\text { Brunner von } \\
\text { Wattenwyl, 1907 }\end{array}$ & RJ, Brasil & NHMW & Ocnophila fortior \\
\hline & O. nattereri & $\begin{array}{c}\text { Brunner von } \\
\text { Wattenwyl, } 1907\end{array}$ & RJ, Brasil & NHMW & Ocnophila nattereri \\
\hline & O. pedestris & $\begin{array}{c}\text { Brunner von } \\
\text { Wattenwyl, } 1907\end{array}$ & Teresópolis, RJ & NHMW & Ocnophila pedestris \\
\hline & O. tuberculata & $\begin{array}{c}\text { Brunner von } \\
\text { Wattenwyl, } 1907\end{array}$ & BA, Brasil & NHMW & Ocnophila tuberculata \\
\hline Oestrophora & O. vittata & Toledo Piza, 1985a & $\begin{array}{c}\text { Boracéia, Salesópolis, } \\
\text { SP }\end{array}$ & ESLQTP & Prexaspes vittata \\
\hline \multirow{3}{*}{ Olcyphides } & O. crassithorax & Toledo Piza, 1937 & Colônia Hansa, SC & ESLQTP & Metriophasma rassithorax \\
\hline & O.dubius & Toledo Piza, 1937 & SP, Brasil & ESLQTP & Perliodes dubius \\
\hline & O. ocellatus & Toledo Piza, 1937 & Blumenau, SC & ESLQTP & Metriophasma ocellatum \\
\hline Otocrania & O. imbe & Toledo Piza, 1939 & Água Preta, BA & ESLQTP & Otocrania imbe \\
\hline Otocraniella & O. flagelloantennata & Zompro, 2004b & $\begin{array}{l}\text { Chapéu do Sol, Serra } \\
\text { do Cipó, MG }\end{array}$ & MZSP & $\begin{array}{c}\text { Otocraniella } \\
\text { flagelloantennata }\end{array}$ \\
\hline \multirow{2}{*}{ Paraphasma } & P. conspersum & Redtenbacher, 1906 & AM, Brasil & NHMW & Paraphasma conspersum \\
\hline & P.paulense & Rehn, 1918 & Cantareira, SP & ANSP & Paraphasma paulense \\
\hline \multirow{2}{*}{ Perliodes } & P. nigrogranulosus & Redtenbacher, 1906 & Teresópolis, RJ & NHMW & Perliodes nigrogranulosus \\
\hline & P. sexmaculatus & Redtenbacher, 1906 & Teresópolis, RJ & NHMW & Perliodes sexmaculatus \\
\hline
\end{tabular}




\begin{tabular}{|c|c|c|c|c|c|}
\hline Gênero & Espécie & Autor/ano & Localidade-tipo & Museu* & Status atual \\
\hline \multirow{8}{*}{ Phantasca } & P.poeciloptera & Günther, 1940 & Fonteboa, AM & MIZ & Pterolibethra Poeciloptera \\
\hline & P. brevipennis & Burmeister, 1838 & Brasil & ZMHB & $\begin{array}{l}\text { Prexaspes (Elasia) } \\
\text { brevipennis }\end{array}$ \\
\hline & P. cneius & Westwood, 1859 & Brasil & BMNH & $\begin{array}{c}\text { Prexapes (Prexaspes) } \\
\text { cneius }\end{array}$ \\
\hline & P. ega & Westwood, 1859 & Brasil & BMNH & Agrostia ega \\
\hline & P. fasciatum & Gray, 1835 & Brasil & $\mathrm{NE}$ & Paraphasma fasciatum \\
\hline & P. flavicorne & Redtenbacher, 1906 & ES, Brasil & NHMW & Pseudophasma flavicorne \\
\hline & P. flavomaculatus & Gray, 1835 & Brasil & BMNH & Anisa flavomaculatus \\
\hline & P. helvolum & Serville, 1838 & Brasil & $\mathrm{NE}$ & Olcyphides helvolus \\
\hline \multirow{14}{*}{ Phasma } & P. hopii & Gray, 1835 & Brasil & BMNH & Olcyphides hopii \\
\hline & P. maculatum & Gray, 1835 & AM, Brasil & BMNH & Paraphasma maculatum \\
\hline & P.paxillus & Westwood, 1859 & Brasil & BMNH & Planudes paxillus \\
\hline & P. phantasma & Westwood, 1859 & Tapajos, AM & BMNH & Phantasca phantasma \\
\hline & P.phlegyas & Westwood, 1859 & Brasil & OXUM & Isagoras phlegyas \\
\hline & P.pholcus & Westwood, 1859 & Santarém, AM & $\mathrm{BMNH}$ & Prexaspes (Elasia) pholcus \\
\hline & P. (Prisopus) horstokkii & Haan, 1842 & Xapuri, AC & BMNH & $\begin{array}{c}\text { Prisopus (Prisopus) } \\
\text { horstokkii }\end{array}$ \\
\hline & P. puppeius & Westwood, 1859 & Tapajos, AM & BMNH & Phantasca puppeius \\
\hline & P. quadratum & Bates, 1865 & $\begin{array}{c}\text { Tapajos, Santarém, } \\
\text { AM }\end{array}$ & OXUM & Paraphasma quadratum \\
\hline & P. servillii & Gray, 1835 & Brasil & $\mathrm{NE}$ & $\begin{array}{c}\text { Prexaspes (Prexaspes) } \\
\text { servillei }\end{array}$ \\
\hline & P. stabilinus & Westwood, 1859 & AM, Brasil & OXUM & Stratocles stabilinus \\
\hline & P. venosum & Burmeister, 1838 & Brasil & ZMHB & Isagoras venosus \\
\hline & P. ditomus & Westwood, 1859 & Brasil & BMNH & Cladoxerus ditomus \\
\hline & P. michaelis & Redtenbacher, 1908. & BA, Brasil & NHMW & Cladomorphus michaelis \\
\hline \multirow{3}{*}{ Phibalosoma } & P.paulense & Toledo Piza , 1938a & São Paulo, SP & ESLQTP & Cladomorphus phyllinus \\
\hline & P. rochai & Toledo Piza, 1938b & CE, Brasil & ESLQTP & Cladomorphus phyllinus \\
\hline & P. phyllocephalum & Westwood, 1859 & Brasil & ZMHB & Otocrania aurita \\
\hline \multirow{2}{*}{ Phthoa } & P. brasiliensis & Toledo Piza, 1938b & Murtinho, MT & ESLQTP & Globocalynda brasiliensis \\
\hline & P. bispinosa & Piza, 1946 & $\begin{array}{l}\text { Porto Cabral, Rio } \\
\text { Paraná, PR }\end{array}$ & MZSP & Phthoa bispinosa \\
\hline \multirow{2}{*}{ Planudes } & P. melzeri & Toledo Piza, 1937 & Bosque da Saúde, SP & ESLQTP & Phthoa melzeri \\
\hline & P. taeniatus & Toledo Piza, 1944 & Ubatuba, SP & MZSP & Phthoa taeniatus \\
\hline Platycrana & P. rugicollis & Gray, 1835 & RJ, Brasil & MVMA & Isagoras rugicollis \\
\hline \multirow{2}{*}{ Prexaspes } & P. (Prexaspes) quadriguttatus & Redtenbacher, 1906 & Obidos, AM & NHMW & $\begin{array}{l}\text { Prexaspes (Prexaspes) } \\
\text { quadriguttatus }\end{array}$ \\
\hline & P. spiniceps & Burmeister, 1838 & Brasil & ZMHB & Prisopus spiniceps \\
\hline \multirow{2}{*}{ Prisopus } & P. spinicollis & Burmeister, 1838 & Brasil & MLUH & Prisopus spinicollis \\
\hline & P. wolfgangjunki & Zompro, 2003 & Brasil & SMTD & Prisopus wolfgangjunki \\
\hline \multirow{2}{*}{ Pseudobacteria } & P. bahiensis & Toledo Piza, 1938a & Jequié, BA & ESLQTP & Phanocloidea bahiensis \\
\hline & P. longipes & Toledo Piza, 1938a & RJ, Brasil & ESLQTP & Paracalynda longipes \\
\hline \multirow[b]{2}{*}{ Pseudophasma } & P. cambridgei & Kirby, 1904 & Santarem, AM & BMNH & Pseudophasma cambridgei \\
\hline & P. septemtrionalis & Toledo Piza, 1977 & PA, Brasil & ESLQTP & $\begin{array}{l}\text { Pseudophasma } \\
\text { septemtrionalis }\end{array}$ \\
\hline Pterolibethra & P. heteronemia & Günther, 1940 & $\begin{array}{c}\text { Rio Negro, São } \\
\text { Gabriel, AM }\end{array}$ & NHRS & Pterolibethra heteronemia \\
\hline \multirow{2}{*}{ Pygirhynchus } & P. carioca & Toledo Piza, 1944 & Rio de Janeiro, RJ & MZSP & Pygirhynchus carioca \\
\hline & P. subfoliatus & Serville, 1838 & Brasil & $\mathrm{NE}$ & Pygirhynchus subfoliatus \\
\hline Reticulonigrum & R. andreaszomproi & Zompro, 2004a & $\begin{array}{l}\text { Santo Antonio do Iça, } \\
\text { AM }\end{array}$ & ZMHB & $\begin{array}{c}\text { Pseudophasma } \\
\text { andreaszomproi }\end{array}$ \\
\hline Stratocles & S. dentatus & Toledo Piza, 1937 & Três Lagos, MT & ESLQTP & Paraphasma dentatum \\
\hline Tersomia & T. brasiliensis & Kirby, 1904 & Iguarassu, $\mathrm{PE}$ & BMNH & Tersomia brasiliensis \\
\hline \multirow{2}{*}{ Xerosoma } & X. canaliculatum & Serville, 1831 & Brasil & MNHN & Xerosoma canaliculatum \\
\hline & $X$. senticosum & Stål, 1875 & BA, Brasil & NHMW & Xerosoma senticosum \\
\hline Xylodus & X. adumbratus & Saussure, 1859 & Brasil & MHNG & Xylodus adumbratus \\
\hline \multirow{2}{*}{ Wattenwylia } & W. cearensis & Toledo Piza, 1938b & CE, Brasil & ESLQTP & Wattenwylia cearensis \\
\hline & $W$. foliata & Toledo Piza, $1938 b$ & CE, Brasil & MZSP & Wattenwylia foliata \\
\hline
\end{tabular}

*NE = Não especificado 


\section{AGRADECIMENTOS}

À Fundação de Amparo a Pesquisa de Minas Gerais (FAPEMIG), pela bolsa de Iniciação Científica concedida para a primeira autora. Aos professores José Paulo Leite Guadanucci e Sebastião Lourenço de Assis Junior pelas críticas nas primeiras versões do manuscrito e a três revisores pelas sugestões que enriqueceram muito este trabalho.

\section{REFERÊNCIAS}

Brock, P.D., Eades, D.C., Otte, D. \& E. Baker, 2011. Phasmida Species File Online (Version 2.1/4.0). Disponível em:

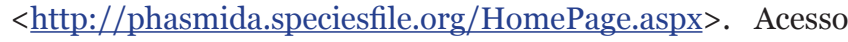
em 01/Jul/ 2012.

Conle, O.\&F.H.Hennemann, 2011. Classification of Phasmatodea. Disponível em: <http://www.phasmatodea.com/web/guest/ classification $>$. Acesso em: 11/Jun/2011.

Crisci, J.V., 2006. One-Dimensional Systematist: Perils in a Time of Steady Progress. Systematic Botany, 31: 217-221.

Kumagai, A.F. \& N.G. Fonseca, 2009. Uma nova espécie de Cladomorphus Gray, 1835 (Phasmatidae, Cladomorphinae) de Minas Gerais, Brasil. Revista Brasileira de Entomologia, 53: 41-44.

Lewinsohn, T.M., A.V.L. Freitas \& P.I. Prado, 2005. Conservação de invertebrados terrestres e seus habitats no Brasil. Megadiversidade, 1: 62- 69.

Lewinsohn, T.M. \& P.I. Prado, 2005. Quantas espécies há no Brasil? Megadiversidade, 1: 36-42.

Marques, A.C. \& C.J.E. Lamas, 2006. Taxonomia zoológica no Brasil: estado da arte, expectativas e sugestões de ações futuras. Papéis Avulsos de Zoologia, 46: 139-174.

Otte, D. \& P. Brock, 2005. Phasmida species file: catalog of stick and leaf insects of the world. Philadelphia, Insect Diversity Association at the Academy of Natural Sciences, 296p.

Savage, J.M., 1995. Systematics and the Biodiversity Crisis. BioScience, 45: 673-679.

Toledo Piza, S., 1936a. Um novo Phasmida do Brasil (Orth.). Revista de Entomologia, 6: 98-100.

Toledo Piza, S., 1936b. Os Phasmidas do Museu Paulista. I. Phasmidae, Bacillinae. Revista de Entomologia, 6: 280-292.

Toledo Piza, S., 1937. Os Phasmidas do Museu Paulista. II. Phasmidae, Phasminae. Revista de Entomologia, 7: 1-8.
Toledo Piza, S., 1938a. Os Phasmidas do Museu Paulista. III. Phasmidae, Cladoxerinae. Revista de Entomologia, 8: 40-44.

Toledo Piza, S., 1938b. Novos Phasmidas do Brasil e da Argentina (Orth.). Revista de Entomologia, 9: 1-11.

Toledo Piza, S., 1939. Dois novos Phasmidas do Brasil (Orth.). Revista de Entomologia, 10: 444-446.

Toledo Piza, S., 1943. Um Proscopiida e um Phasmida novos do Brasil (Orth.). Revista de Entomologia, 14: 287-291.

Toledo Piza, S., 1944. Cinco novas espécies de Phásmidas do Brazil. Anais da Escola Superior de Agricultura "Luiz de Queiroz". Separata, 3:43-58.

Toledo Piza, S., 1977. Três novas espécies de Pseudophasma (Phasmatodea - Phyllidae). Anais da Escola Superior de Agricultura "Luiz de Queiroz", 34: 489-491.

Toledo Piza, S., 1985a. A segunda espécie do gen. Oestrophora (Phasmatodea: Phylliidae). Revista de Agricultura, 60: 1-2.

Toledo Piza, S., 1985b. Um novo Stratocles do Brasil (Phasmatodea: Phylliidae). Revista de Agricultura, 60: 101102.

Wheeler, Q.D., 2007. Invertebrate systematics or spineless taxonomy? Zootaxa, 1668: 11-18.

Zompro, O. \& J. Adis. 2001. A new species of Phasmatodea of the genus Echetlus Stål. Revista de Agricultura 76: 291-297.

Zompro, O., 2004a. Revision of the genera of the Areolatae, including the status of Timema and Agathemera (Insecta: Phasmatodea). Abhandiungen des Naturwissenschaftlichen Vereins in Hamburg, 37: 1-327.

Zompro, O., 2004b. A Key to the Stick-Insect Genera of the 'Anareolatae' of the New World, with Descriptions of Several New Taxa (Insecta: Phasmatodea). Studies on Neotropical Fauna and Environment, 39: 133-144.

Zompro, O \& F.C. Domenico, 2005. Catalogue of the type material of Phasmatodea (Insecta) depositedin Brazilian Museums. Iheringia, 95: 255-259.

Recebido em: 24/o1/2012

Aceito em: 22/o9/2012

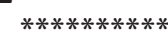

\section{Como citar este artigo:}

Araujo, F.F \& A.R.S.Garraffoni, 2012. Sinopse dos Phasmatodea (Insecta) Descritos para o Brasil. EntomoBrasilis, 5(3): 232-237.

Acessível em: http://www.periodico.ebras.bio.br/ojs/index.php/ebras/article/view/229

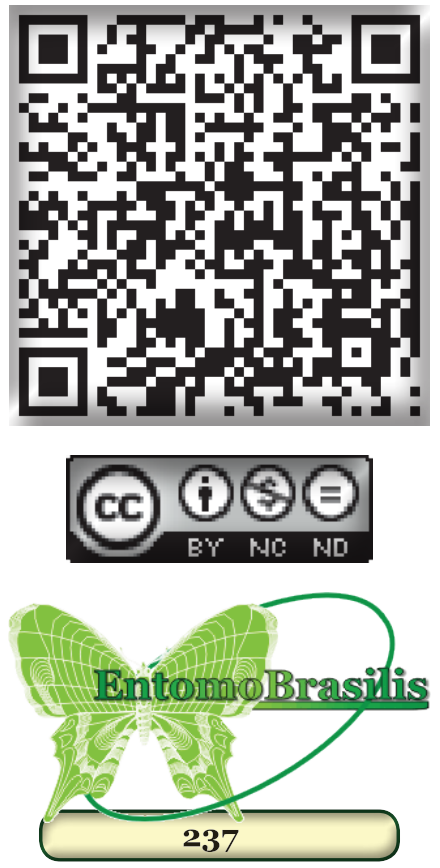

